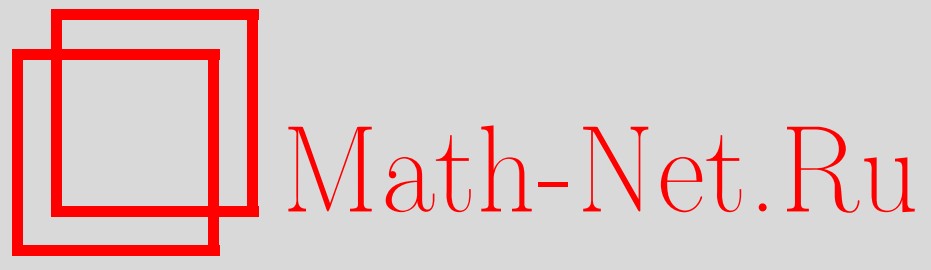

M. Weber, On the CLT for means under the rotation action. I, Теория вероятн. и ее примен., 2005, том 50, выпуск 4, 733-753

DOI: https://doi.org/10.4213/tvp127

Использование Общероссийского математического портала Math-Net.Ru подразумевает, что вы прочитали и согласны с пользовательским соглашением

http://www . mathnet.ru/rus/agreement

Параметры загрузки:

IP : 54.162 .27 .143

26 апреля 2023 г., 15:21:46

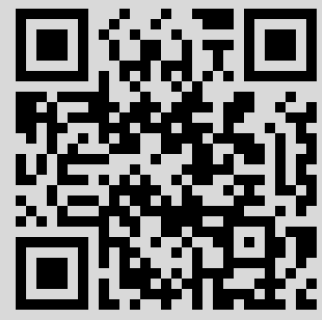




\section{ON THE CLT FOR MEANS UNDER THE ROTATION ACTION. I}

Предлагается метод, позволяющий построить для различных типичных средних, порожденных действием любого иррационального вращения круга, примеры $L^{2}$-функций, удовлетворяющих центральной предельной теореме. В качестве примера рассматриваются нелинейные средние и средние по последовательности квадратов. Во втором случае используется метод круга Харди-Литтвуда. Мы также даем пример непрерывного случайного гауссовского ряда Фурье, траектории которого удовлетворяют как центральной предельной теореме, так и центральной предельной теореме в смысле сходимости почти наверное.

Ключевые слова и фразы: центральная предельная теорема с вероятностью единица, иррациональные вращения, нелинейные средние, квадратичные средние, взвешенные средние, гауссовская рандомизация, случайные ряды Фурье, метод круга.

1. Introduction-results. Let $(X, \mathscr{A}, \mu, \tau)$ be a measurable dynamical system. By Birkhoff's pointwise ergodic theorem, we know that for any $f \in L^{1}(\mu)$, the limit

$$
\lim _{n \rightarrow \infty} \frac{1}{n} \sum_{k=0}^{n-1} f \circ \tau^{k} x=\bar{f}(x)
$$

exists $\mu$-almost everywhere and in $L^{1}(\mu)$, and we have $\bar{f}=\mathbf{E}\{f \mid \mathscr{J}\}$, where $\mathscr{J}=\sigma\left\{A \in \mathscr{A}: \tau^{-1} A=A\right\}$. A parallel result in probability theory is the well-known strong law of large numbers asserting that if $X, X_{1}, X_{2}, \ldots$ are independent identically distributed (i.i.d.) integrable random variables with basic probability space $(\Omega, \mathscr{B}, \mathbf{P})$ and partial sums $S_{n}=X_{1}+\cdots+X_{n}$, then

$$
\mathbf{P}\left\{\lim _{n \rightarrow \infty} \frac{S_{n}}{n}=\mathbf{E} X\right\}=1 .
$$

Recall that the strong law of large numbers is just a very particular case of the pointwise ergodic theorem; and even in the case of sequences of i.i.d. random variables, the pointwise ergodic theorem expresses a much more

* Mathématique (IRMA), Université Louis-Pasteur et C.N.R.S., 7, rue René Descartes, 67084 Strasbourg Cedex, France; e-rnail: weber@math.u-strasbg.fr 
stronger property. As is well known, the strong law of large numbers is completed by two fundamental results: the law of the iterated logarithm and the central limit theorem. This last result, which concerns the statistic of this convergence, states under the assumptions $\mathbf{E} X=0, \mathbf{E} X^{2}=1$ that

$$
\lim _{n \rightarrow \infty} \frac{S_{n}}{\sqrt{n}} \stackrel{\mathscr{D}}{=} N(0,1)
$$

In the late eighties, a companion to this result were found independently by Brosamler, Fisher, and Schatte. Following the formulation given in [13], we have, under the same assumptions,

$$
\lim _{N \rightarrow \infty} \frac{1}{\ln N} \sum_{j=1}^{N} \frac{1}{j} \delta_{\left\{S_{j} / \sqrt{j}\right\}} \stackrel{\mathscr{D}}{=} N(0,1) .
$$

Much stronger forms of this result can be however obtained by using the notion of quasi-orthogonal system of functions (see [6]-[8]).

It is natural to ask whether similar results hold for dynamical systems, and under which conditions. Such questions were, and are still intensively investigated; see, for instance, the survey paper [4]. One can cite Kac [10] as an initiator of investigations on the central limit theorem in dynamical systems. The fundamental result is certainly the theorem of Burton and Denker [3], which asserts the existence a real-valued function $f$, defined on the phase space $X$ of any given aperiodic dynamical system $(X, \mathscr{A}, \mu, T)$, such that the natural long-term ratio satisfies the central limit theorem:

$$
\mu\left\{x \in X: \frac{\sum_{j=0}^{n-1} T^{j} f(x)}{\left\|\sum_{j=0}^{n-1} T^{j} f\right\|_{2}} \leqslant u\right\} \rightarrow \frac{1}{\sqrt{2 \pi}} \int_{-\infty}^{u} e^{-v^{2} / 2} d v
$$

as $n \rightarrow \infty$, for all $u \in \mathbf{R}$. The original proof of (1.5), however, contained a mistaken argument which has been amended in [5]. The Burton-Denker result was also recently completed by a fine Gaussian approximation type result in [19].

In the present work, we will focus on a particular, but fundamental type of dynamical systems: the irrational rotations of the circle. Existence results of type (1.5) in the case of rotations where already obtained in [3] and [11]. Note that, in view of the conjugacy lemma, this already implies the same type of results for a weak dense class of automorphisms. For usual ergodic means, we can also mention a delicate dichotomy type result proved in [12].

Let $X=\mathbf{R} / \mathbf{Z}=[0,1[$ denote the circle with normalized Lebesgue's measure $m$, and let $\theta \in] 0,1\left[\cap \mathbf{Q}^{c}\right.$ with associated rotation $\tau x=x+\theta$ $(\bmod 1), x \in X$. We will study the central limit theorem (CLT) and almost sure central limit theorem (ASCLT) for various ergodic sums generated by the action of $\tau$, and also the asymptotic behavior of the CLT-normalized 
partial sums. We propose a construction of functions satisfying the CLT and will treat, apart from the usual ergodic sums $S_{N}^{\tau}(f)(t)=\sum_{k=0}^{N-1} f\left(\tau^{k} t\right)$, three typical examples:

(A) nonlinear ergodic sums: $S_{N}^{\tau}(f, g)(t)=\sum_{k=0}^{N-1} f\left(\tau^{k} t\right) g\left(\tau^{2 k} t\right)$,

(B) weighted ergodic sums: $U_{N}^{\tau}(f)(t)=\sum_{k=0}^{N-1} \sigma_{k} f\left(\tau^{k} t\right)$,

(C) ergodic sums along the squares: $\Gamma_{N}^{\tau}(f)(t)=\sum_{k=0}^{N-1} f\left(\tau^{k^{2}} t\right)$.

The principle of the construction can be described as follows: let $\left\{\left[a_{j}, b_{j}[, j \geqslant 1\}\right.\right.$ be some sequence of nonoverlapping intervals with $\lim _{j \rightarrow \infty} a_{j}=\lim _{j \rightarrow \infty} b_{j}=0$. Let $\mathscr{L}=\left\{l_{j}, j \geqslant 1\right\}$ be a lacunary sequence verifying $\left\{l_{j} \theta\right\} \in\left[a_{j}, b_{j}\right.$ [ for any $j \geqslant 1$. For an appropriate choice of the intervals $\left[a_{j}, b_{j}[\right.$, mild assumptions on the Fourier coefficients of $f$ will ensure that $f$ satisfies the CLT (Theorem 5.1). Analogous results are derived for sample paths of random Fourier series. In Theorem 6.2, an example with continuous sample paths satisfying both CLT and ASCLT is given.

In complement to that study we examine in Section 3 for a class of random Fourier series the behavior of CLT-normalized partial sums. The results obtained allow, when combined with Berkes's result [1] and Schur's theorem for quasi-orthogonal systems, to provide nontrivial examples of functions $f \in L^{2}(m)$ possessing strong uniform approximations properties either by i.i.d. random variables or orthonormal sequences regarding their normalized partial sums (see Section 4).

The paper is organized as follows. In Section 2, the construction is described and a standard decomposition of partial sums is presented. Section 3 is devoted to the study of the oscillations properties of sub-Gaussian random Fourier series. Section 4 concerns examples of well-approximable normalized partial sums. In Section 5, a vector space of functions satisfying the CLT for $\tau$ is determined, next conditions are obtained for the sample paths of a Gaussian random Fourier series to satisfy the CLT under the action of $\tau$. Some examples are given. In Section 6, we show how to adapt the construction to prove the CLT for the cases (A), (B), and (C). In Section 7, the ASCLT is examined for Gaussian random Fourier series in relation with the CLT.

2. Notation-construction. Let $f: X \rightarrow \mathbf{R}$ be Lebesgue measurable and put for any integer $N \geqslant 1: S_{N} f=S_{N}^{\tau} f=\sum_{k=0}^{N-1} f \circ \tau^{k}$. We say that a function $f \in L^{2}(m)$ satisfies the CLT when $S_{N} f /\left\|S_{N} f\right\|_{2} \stackrel{\mathscr{D}}{\Longrightarrow} N(0,1)$; and that $f$ satisfies the ASCLT when $(1 / \ln N) \sum_{n=1}^{N} k^{-1} \delta_{\left\{S_{N} f /\left\|S_{N} f\right\|_{2}\right\}} \stackrel{\mathscr{D}}{\Longrightarrow}$ $N(0,1)$. We thus study the CLT for the set of ergodic means discussed in the previous section, in the sense of [3], see also [4].

Throughout the paper, the letter $C$ (respectively, $C_{\alpha, \beta, \ldots}$ ) will denote some universal constant (respectively, some constant depending on $\alpha, \beta, \ldots$ only) which may possibly change of value at each occurrence. We first operate a construction adapted to the study of the usual sums. 
Let first $\varepsilon=\left(\varepsilon_{n}\right)_{n \geqslant 1}$ be some sequence of reals in ]0,1] decreasing to 0 . To the sequence $\varepsilon$ we associate an increasing sequence of positive integers $\mathscr{N}=\left(N_{j}\right)_{j \geqslant 1}$ defined as follows:

$$
N_{1} \geqslant 1 \quad \text { is arbitrary, and for } n \geqslant 2, \quad N_{n}=\left\lfloor\frac{16^{n-1} N_{1}}{\varepsilon_{n} \varepsilon_{n-1}^{2} \cdots \varepsilon_{2}^{2} \varepsilon_{1}}\right\rfloor .
$$

Next we associate to $\theta$ and sequence $\varepsilon$ another increasing sequence of positive integers $\mathscr{L}=\left(l_{j}\right)_{j \geqslant 1}$ as follows: for any $n \geqslant 1, l_{n}$ is chosen so that

$$
\frac{\varepsilon_{1} \varepsilon_{2}^{2} \cdots \varepsilon_{n}^{2}}{2 \cdot 16^{n} N_{1}} \leqslant\left\{l_{n} \theta\right\} \leqslant \frac{\varepsilon_{1} \varepsilon_{2}^{2} \cdots \varepsilon_{n}^{2}}{16^{n} N_{1}} .
$$

This is possible since the sequence $(\{n \theta\})_{n \geqslant 1}$ is everywhere dense in $[0,1]$. For later use, it is worthwhile to indicate that given any sequence $\eta=\left(\eta_{n}\right)_{n \geqslant 1}$ decreasing to 0 , we may (and will do later on) choose sequence $\mathscr{L}$ to satisfy (2.2) and

$$
\forall n \geqslant 1, \quad \frac{l_{n}}{l_{n+1}} \leqslant \eta_{n} .
$$

The sequence $\mathscr{N}$ is used to define a partition of $\mathbf{N}$ with blocks $I_{j}:=$ $\left[N_{j}, N_{j+1}[, j \geqslant 1\right.$, on which the oscillations of partials sums are estimated. The sequence $\mathscr{L}$ will serve to define the support (in $L^{2}(m)$ ) of our random Fourier series. Let $\beta=\left(\beta_{k}\right)_{k \in \mathbf{N}} \in \ell_{2}$ with $\beta_{0}=0, \beta_{n}=\beta_{-n}>0$ for $n \neq 0$ and $\sum_{k=1}^{\infty} \beta_{k}^{2}=1$. Let $e(t)=\exp (2 i \pi t), e_{n}(t)=e(n t), n \in \mathbf{Z}$. We define

$$
f(t)=\frac{1}{2} \sum_{k \in \mathbf{Z}} \beta_{k} e_{l_{k}}(t)=\sum_{k=1}^{\infty} \beta_{k} \cos 2 \pi l_{k} t .
$$

Let $g=\left(g_{k}\right)_{k \in \mathbf{N}}, g^{\prime}=\left(g_{k}^{\prime}\right)_{k \in \mathbf{N}}$ be two independent sequences of i.i.d. random variables with basic probability space $(\Omega, \mathscr{B}, \mathbf{P})$ (different from $(X, \mathscr{A}, m)$ ), and such that $\mathbf{E} g_{k}=\mathbf{E} g_{k}^{\prime}=0$ and $\mathbf{E} g_{k}^{2}=\mathbf{E} g_{k}^{\prime 2}=1$. We assume that $g_{k}, g_{k}^{\prime}$ are sub-Gaussian random variables. We will consider the associated sequence $\gamma=\left(\gamma_{k}\right)_{k \in \mathbf{N}}$, where $\gamma_{k}=g_{k}+i g_{k}^{\prime}$, of complex-valued random variables. We define the random Fourier series associated to $f$ as follows:

$$
\mathscr{X}(t)=\mathscr{X}_{f}(t)=\sum_{k \in \mathbf{N}} \beta_{k} \operatorname{Re}\left(\gamma_{k} \overline{l_{k}}(t)\right)=\sum_{k=1}^{\infty} \beta_{k}\left[g_{k} \cos 2 \pi l_{k} t+g_{k}^{\prime} \sin 2 \pi l_{k} t\right] .
$$

Next we put for any integer $N \geqslant 1$ and real $\theta \in[-1,1]$

$$
V_{N}(\theta)=\frac{1}{N} \sum_{k=0}^{N-1} e_{k}(\theta)=\frac{e_{N}(t)-1}{N(e(t)-1)} .
$$


Then, for any integer $N$

$$
\begin{aligned}
S_{N}(f)(t) & =N \sum_{k=1}^{\infty} \beta_{k} \operatorname{Re}\left[V_{N}\left(l_{k} \theta\right)\right] \cos 2 \pi l_{k} t, \\
S_{N}\left(X_{f}\right)(t) & =\sum_{k=1}^{\infty} \beta_{k} \operatorname{Re}\left[\gamma_{k} \overline{e_{l_{k}}(t) V_{N}\left(l_{k} \theta\right)}\right] .
\end{aligned}
$$

We shall frequently use the following estimates valid for any integer $n \geqslant 1$ and depending on the choice of sequences $\mathscr{N}$ and $\mathscr{L}$ :

$$
\begin{aligned}
\left(\mathscr{L}_{n}\right) & \forall N \leqslant N_{n}, & \left|V_{N}\left(\left\{l_{n} \theta\right\}\right)-1\right| \leqslant 2 \varepsilon_{n}, \\
\left(\mathscr{N}_{n}\right) & \forall N \geqslant N_{n}, \quad \forall m<n, & \left|V_{N}\left(\left\{l_{m} \theta\right\}\right)\right| \leqslant 2 \varepsilon_{n} .
\end{aligned}
$$

These ones are easily deduced from standard estimates of the kernels $V_{N}$ : for any integer $N \geqslant 1$ and $y \in[-1,1]$,

$$
\left|V_{N}(y)-1\right| \leqslant 32 N y, \quad\left|V_{N}(y)\right| \leqslant \frac{1}{2 N y} \text {. }
$$

Then, on the one hand $\left(N \leqslant N_{n}\right)$,

$$
\begin{aligned}
\left|V_{N}\left(\left\{l_{n} \theta\right\}\right)-1\right| & \leqslant 32 N\left\{l_{n} \theta\right\} \leqslant 32 N_{n}\left\{l_{n} \theta\right\} \\
& \leqslant 32 \frac{16^{n-1} N_{1}}{\varepsilon_{n} \varepsilon_{n-1}^{2} \cdots \varepsilon_{2}^{2} \varepsilon_{1}} \frac{\varepsilon_{1} \varepsilon_{2}^{2} \cdots \varepsilon_{n}^{2}}{2 \cdot 16^{n} N_{1}}=2 \varepsilon_{n},
\end{aligned}
$$

and on the other $\left(N \geqslant N_{n}, n>m\right)$,

$$
\left|V_{N}\left(\left\{l_{m} \theta\right\}\right)\right| \leqslant \frac{1}{2 N\left\{l_{m} \theta\right\}} \leqslant \frac{1}{2 N_{n}\left\{l_{m} \theta\right\}} \leqslant \frac{\varepsilon_{n} \varepsilon_{n-1}^{2} \cdots \varepsilon_{2}^{2} \varepsilon_{1}}{2\left(\frac{1}{2}\right) 16^{n-1} N_{1}} \frac{2 \cdot 16^{m} N_{1}}{\varepsilon_{1} \varepsilon_{2}^{2} \cdots \varepsilon_{m}^{2}} \leqslant 2 \varepsilon_{n} .
$$

Decomposition of partial sums. The following decomposition of partials sums $S_{N}\left(\mathscr{X}_{f}\right)(t)$ is essential and will allow to control the asymptotic behavior of these ones, in particular the study of their oscillation function around a sequence of rests of some random series. This will be undertaken in the next section. In order to avoid unnecessary heavy notation, we shall simply write throughout the rest of the paper

$$
S_{N}=S_{N}\left(\mathscr{X}_{f}\right)(t),
$$

unless explicited. The decomposition of $S_{N}$ goes as follows; we put for positive integers $N$ and $j$ :

$$
\begin{array}{lll}
S_{N}=\check{S}_{N}+\widehat{S}_{N}, & \text { where } & \check{S}_{N}=N \sum_{k>j} \beta_{k} \operatorname{Re}\left[\gamma_{k} \overline{e_{l_{k}} V_{N}\left(l_{k} \theta\right)}\right] \\
\check{S}_{N}=S_{N}^{\prime}+s_{N}, & \text { where } & S_{N}^{\prime}=N \sum_{k<j} \beta_{k} \operatorname{Re}\left[\gamma_{k} \overline{\left.e_{l_{k}} V_{N}\left(l_{k} \theta\right)\right)}\right] \\
T_{N}=N \sum_{k>j} \beta_{k} \operatorname{Re}\left[\gamma_{k} \overline{e_{k}}\right], & b_{j}=\left(\sum_{k>j}^{\infty} \beta_{k}^{2}\right)^{1 / 2} .
\end{array}
$$


We observe for $N \in I_{j}$ that $T_{N} /\left(N b_{j}\right)$ depend on $j$ only, and is equal to

$$
\Theta_{j}:=\frac{\sum_{k>j} \beta_{k} \operatorname{Re}\left(\gamma_{k} \overline{e_{l_{k}}}\right)}{b_{j}} .
$$

For any $N \in I_{j}, j \geqslant 1$, put $\tau_{N}=N b_{j}$. As will be seen later on, mild assumptions on the sequences $\varepsilon$ and $\beta$ will ensure that

$$
\mathbf{P}\left\{\lim _{N \rightarrow \infty} \frac{\left\|S_{N}\right\|_{2, m}}{\tau_{N}}=1\right\}=1 .
$$

We also put

$$
Y_{N}=\frac{\check{S}_{N}-T_{N}}{\tau_{N}}, \quad Z_{N}=\frac{S_{N}^{\prime}}{\tau_{N}}, \quad U_{N}=\frac{\widehat{S}_{N}-S_{N}^{\prime}}{\tau_{N}} .
$$

Then, for $N \in I_{j}$, we have the relation $S_{N} / \tau_{N}-\Theta_{j}=Y_{N}+Z_{N}+U_{N}$.

Estimation of norms. Put $\zeta_{k}=\overline{e_{l_{k}}} N\left(\overline{V_{N}\left(l_{k} \theta\right)}-1\right)=z_{k}+i z_{k}^{\prime}$. Then $\operatorname{Re}\left(\gamma_{k} \zeta_{k}\right)=g_{k} z_{k}-g_{k}^{\prime} z_{k}^{\prime}$, and thus

$$
\check{S}_{N}-T_{N}=\sum_{k>j} \beta_{k}\left(g_{k} z_{k}-g_{k}^{\prime} z_{k}^{\prime}\right) .
$$

Hence

$$
\left\|\check{S}_{N}-T_{N}\right\|_{2, \mathbf{P}}^{2}=\sum_{k: k \geqslant j+1} \beta_{k}^{2}\left(\left(z_{k}\right)^{2}+\left(z_{k}^{\prime}\right)^{2}\right)=\sum_{k: k \geqslant j+1} \beta_{k}^{2} N^{2}\left|V_{N}\left(l_{k} \theta\right)-1\right|^{2} .
$$

As $N \in I_{j}$ and $k>j$, we have $N<N_{k}$ and by property $\left(\mathscr{L}_{k}\right),\left|V_{N}\left(l_{k} \theta\right)-1\right| \leqslant$ $2 \varepsilon_{k}$. Thus,

$$
\left\|\check{S}_{N}-T_{N}\right\|_{2, \mathbf{P}}^{2} \leqslant 4 N^{2} \sum_{k>j} \beta_{k}^{2} \varepsilon_{k}^{2} \leqslant 4 \varepsilon_{j+1}^{2} N^{2} \sum_{k>j} \beta_{k}^{2}=4 \varepsilon_{j+1}^{2} b_{j}^{2} N^{2} .
$$

Hence for any $j \geqslant 1$ and $n \in I_{j}$,

$$
\left\|\check{S}_{N}-T_{N}\right\|_{2, \mathbf{P}} \leqslant 2 \varepsilon_{j+1} b_{j} N \text { and }\left\|Y_{N}\right\|_{2, \mathbf{P}} \leqslant 2 \varepsilon_{j+1} .
$$

Besides, by property $\left(\mathscr{N}_{j}\right)$,

$$
\left\|S_{N}^{\prime}\right\|_{2, \mathbf{P}}^{2}=\sum_{k<j} \beta_{k}^{2} N^{2}\left|V_{N}\left(l_{k} \theta\right)\right|^{2} \leqslant 4 \sum_{k<j} \beta_{k}^{2} N^{2} \varepsilon_{j}^{2} \leqslant 4 N^{2} \varepsilon_{j}^{2},
$$

since $k<j$ and $N \geqslant N_{j}$. Thus, for any $j \geqslant 1$ and $N \in I_{j}$,

$$
\left\|S_{N}^{\prime}\right\|_{2, \mathbf{P}} \leqslant 2 \varepsilon_{j} N \quad \text { and } \quad\left\|Z_{N}\right\|_{2, \mathbf{P}} \leqslant \frac{2 \varepsilon_{j}}{b_{j}} .
$$


The perturbating term is the reminding quantity $s_{N}=\beta_{j} N \operatorname{Re}\left[\gamma_{j} \overline{e_{l_{j}} V_{N}\left(l_{j} \theta\right)}\right]$, for $N \in I_{j}$. In that case $\left|V_{N}\left(l_{j} \theta\right)\right|$ remains undetermined. We have for all $j \geqslant 1$ and $N \in I_{j}$,

$$
\left\|s_{N}\right\|_{2, \mathbf{P}} \leqslant \beta_{j} N \quad \text { and } \quad\left\|U_{N}\right\|_{2, \mathbf{P}} \leqslant \frac{\beta_{j}}{b_{j}} .
$$

In general the series $\sum_{j}\left(\beta_{j} / b_{j}\right)^{2}$ diverges, but we may choose sequence $\beta$ so that the series $\sum_{j}\left(\beta_{j} / b_{j}\right)^{a}$ converges for $a>2$. This fact will impose the choice of some Orlicz's spaces in which we may well analyse the oscillation of partial sums $S_{N}$ around $\Theta_{j}$ as will be seen again in the next section. We close this one by indicating the following estimate which is plainly derived from the three previous ones:

$$
\left\|\frac{S_{N}}{\tau_{N}}-\Theta_{j}\right\|_{2, \mathbf{P}} \leqslant 4 \frac{\varepsilon_{j}}{b_{j}}+\frac{\beta_{j}}{b_{j}} .
$$

This bound clearly shows a limit in the choice of the sequence $\beta$; this one cannot grow more than geometrically.

3. Oscillations of partial sums. This section concerns the study of the oscillation of normalized partial sums $S_{N} / \tau_{N}$ around $\Theta_{j}$ over the blocks $I_{j}$. Let $r>2$ and introduce the oscillation function

$$
W_{r}=\left\{\sum_{j=1}^{\infty} \sup _{N \in I_{j}}\left|\frac{S_{N}}{\tau_{N}}-\Theta_{j}\right|^{r}\right\}^{1 / r} .
$$

Put

$$
\Delta_{r}=\left(\sum_{j=1}^{\infty}\left\{\frac{\varepsilon_{j}}{b_{j}}\left[\sum_{k=1}^{j+1} \ln \frac{1}{\varepsilon_{k}}\right]^{1 / 2}\right\}^{r}\right)^{1 / r}+\left(\sum_{j=1}^{\infty}\left[\frac{\beta_{j}}{b_{j}}\right]^{r}\right)^{1 / r}
$$

The following proposition is the main result of this section.

Proposition 3.1. For any $r>2$,

$$
\left\|W_{r}\right\|_{G, \nu} \leqslant C_{r} \Delta_{r}
$$

$\mathrm{P} \mathrm{r}$ o o f. It will be convenient to work with the following quantities:

$$
\begin{aligned}
& \mathscr{Y}^{j}=\sup _{N \in I_{j}}\left|Y_{N}\right|^{r}, \quad \mathscr{Z}^{j}=\sup _{N \in I_{j}}\left|Z_{N}\right|^{r}, \quad \mathscr{U}^{j}=\sup _{N \in I_{j}}\left|U_{N}\right|^{r}, \\
& \mathscr{Y}_{r}=\left(\sum_{j=1}^{\infty} \mathscr{Y}^{j}\right)^{1 / r}, \quad \mathscr{Z}_{r}=\left(\sum_{j=1}^{\infty} \mathscr{Z}^{j}\right)^{1 / r}, \quad \mathscr{U}_{r}=\left(\sum_{j=1}^{\infty} \mathscr{U}^{j}\right)^{1 / r} .
\end{aligned}
$$

According to the decompostion $S_{N} / \tau_{N}-\Theta_{j}=Y_{N}+Z_{N}+U_{N}$, we have

$$
W_{r} \leqslant C_{r}\left(\mathscr{Y}_{r}+\mathscr{Z}_{r}+\mathscr{U}_{r}\right) .
$$


Put for any $s>0, G_{s}(x)=e^{|x|^{s}}-\sum_{n s<1}|x|^{n s} / n !, x \in \mathbf{R}$, with the writing convention: $G_{2}(x)=G(x)$. Let $(D, \mu)$ be some probability space and $L^{G_{s}}(\mu)$ be the associated Orlicz space with norm defined by

$$
\|h\|_{G_{s}, \mu}=\inf \left\{\alpha>0: \int_{D} G_{s}\left(\frac{h}{\alpha}\right) d \mu \leqslant 1\right\} \quad\left(h \in L^{0}(\mu)\right) .
$$

Let also $M_{r}<\infty$ be such that $e^{|x|^{s}} \leqslant 2\left(G_{s}(x)+1\right)$ for $|x| \geqslant M_{r}$. Since $g$ and $g^{\prime}$ are sub-Gaussian random variables, $Y_{N}, Z_{N}, U_{N}$ are sub-Gaussian too and belong to $L^{G}(\mathbf{P})$. Since for any finite index $I$ :

$$
\left\|\sup _{i \in I}\left|f_{i}\right|\right\|_{G, \nu} \leqslant\left(\frac{2}{\ln 2}\right)^{1 / 2} \sup _{i \in I}\left\|f_{i}\right\|_{G, \nu} \sqrt{\sharp(I)}
$$

we have for all $t \in X$, by using estimate (2.8)

$$
\begin{aligned}
\left\|\left(\mathscr{Y}^{j}\right)^{1 / r}(t)\right\|_{G, \mathbf{P}} & =\left\|\sup _{N \in I_{j}}\left|Y_{N}(t)\right|\right\|_{G, \mathbf{P}} \leqslant C \sup _{N \in I_{j}}\left\|Y_{N}(t)\right\|_{2, \mathbf{P}} \sqrt{\ln \sharp\left(I_{j}\right)} \\
& \leqslant C \varepsilon_{j+1} \sqrt{\ln \sharp\left(I_{j}\right)} .
\end{aligned}
$$

This implies that $\left\|\left(\mathscr{Y}^{j}\right)^{1 / r}\right\|_{G, \nu} \leqslant C \varepsilon_{j+1} \sqrt{\ln \sharp\left(I_{j}\right)}$. Therefore,

$$
\int_{X \times \Omega} \exp \left(\left[\frac{\left(\mathscr{Y}^{j}\right)^{1 / r}}{C \varepsilon_{j+1} \sqrt{\ln \sharp\left(I_{j}\right)}}\right]^{2}\right) d \nu \leqslant 2 .
$$

Consequently,

$$
\int_{X \times \Omega} G_{2 / r}\left(\frac{\mathscr{Y}^{j}}{\left[C \varepsilon_{j+1} \sqrt{\ln \sharp\left(I_{j}\right)}\right]^{r}}\right) d \nu \leqslant 1 .
$$

Hence, $\left\|\mathscr{Y}^{j}\right\|_{G_{2 / r}, \nu} \leqslant C_{r}\left(\varepsilon_{j+1} \sqrt{\ln \sharp\left(I_{j}\right)}\right)^{r}$. Since $\mathscr{Y}_{r}^{r}=\sum_{j=1}^{\infty} \mathscr{Y}^{j}$, we deduce by means of the triangle inequality $\left\|\mathscr{Y}_{r}^{r}\right\|_{G_{2 / r}, \nu} \leqslant C_{r} \sum_{j=1}^{\infty}\left(\varepsilon_{j+1} \sqrt{\ln \sharp\left(I_{j}\right)}\right)^{r}$. We denote by $B$ the bound obtained. Then

$$
\int_{X \times \Omega} G_{2 / r}\left(\frac{\mathscr{Y}_{r}^{r}}{B}\right) d \nu \leqslant 1
$$

But

$$
\begin{aligned}
& \int_{X \times \Omega} \exp \left(\left(\frac{\mathscr{Y}_{r}}{B \frac{1}{r}}\right)^{2}\right) d \nu=\int_{X \times \Omega} \exp \left(\left(\frac{\mathscr{Y}_{r}^{r}}{B}\right)^{2 / r}\right) d \nu \\
& =\left\{\int_{\mathscr{Y}_{r}^{r} / B<M_{r}}+\int_{\mathscr{Y}_{r}^{r} / B} \geqslant M_{r}\right\} \exp \left(\left(\frac{\mathscr{Y}_{r}^{r}}{B}\right)^{2 / r}\right) d \nu \\
& \leqslant \exp \left(M_{r}^{2 / r}\right)+2 \int_{X \times \Omega}\left[G_{2 / r}\left(\frac{\mathscr{Y}_{r}^{r}}{B}\right)+1\right] d \nu \leqslant \exp \left(M_{r}^{2 / r}\right)+4 .
\end{aligned}
$$


It follows that

$$
\left\|\mathscr{Y}_{r}\right\|_{G, \nu} \leqslant C_{r}\left(\sum_{j=1}^{\infty}\left[\varepsilon_{j+1} \sqrt{\ln \sharp\left(I_{j}\right)}\right]^{r}\right)^{1 / r} .
$$

Using now estimate (2.9) and arguing similarly, we get

$$
\left\|\mathscr{Z}_{r}\right\|_{G, \nu} \leqslant C_{r}\left(\sum_{j=1}^{\infty}\left[\frac{\varepsilon_{j}}{b_{j}} \sqrt{\ln \sharp\left(I_{j}\right)}\right]^{r}\right)^{1 / r} .
$$

Finally, estimate (2.10) together with the same line of arguments, also leads to

$$
\left\|\mathscr{U}_{r}\right\|_{G, \nu} \leqslant C_{r}\left(\sum_{j=1}^{\infty}\left[\frac{\beta_{j}}{b_{j}}\right]^{r}\right)^{1 / r}
$$

Notice now that $\ln \sharp\left(I_{j}\right) \leqslant \ln N_{j+1} \leqslant C \sum_{k=1}^{j+1} \operatorname{in}\left(1 / \varepsilon_{k}\right)$. Since $W_{r} \leqslant C_{r}\left(\mathscr{Y}_{r}+\right.$ $\mathscr{Z}_{r}+\mathscr{U}_{r}$ ), we have obtained

$$
\left\|W_{r}\right\|_{G, \nu} \leqslant C_{r} \Delta_{r}
$$

This achieves the proof of the proposition.

$\mathrm{R}$ e $\mathrm{m}$ a $\mathrm{r} \mathrm{k}$ 3.1. From the very proof of Proposition 3.1 it follows that the conditions

$$
\lim _{j \rightarrow \infty} \frac{\beta_{j}}{b_{j}} \sqrt{\ln j}=0, \quad \sum_{j \geqslant 1} \frac{\varepsilon_{j}^{2} \ln N_{j+1}}{b_{j}^{2}}<\infty
$$

are sufficient to imply

$$
\mathbf{P}\left\{\lim _{j \rightarrow \infty} \sup _{N \in I_{j}}\left|\frac{S_{N}}{\tau_{N}}-\Theta_{j}\right|=0\left(\text { in } L^{2}(m) \text { and } m \text {-a.e. }\right)\right\}=1 .
$$

4. Uniform approximation by i.i.d. random variables. Let us assume that sequence $\beta$ is a sequence of positive reals with $\sum_{j} \beta_{j}=\infty$ and $\sum_{j} \beta_{j}^{2}<\infty$. It is also further assumed that sequences $\varepsilon$ and $\beta$ are linked by the condition $\Delta_{r}<\infty$ for some $r>2$ (see (3.2)). We apply Proposition 3.1 in the case when both sequences $g, g^{\prime}$ are Rademacher's sequences to obtain a theorem of existence of $L^{2}$ functions for which partial sums of the CLT satisfy a strong uniform approximation by i.i.d. random variables. Put

$$
\begin{aligned}
\mathscr{X}_{f}^{\prime}(\omega, t) & =\frac{\mathscr{X}_{f}(\omega, t)+\mathscr{X}_{f}(\omega, 2 \pi-t)}{2}, \\
\Theta_{j}^{\prime}(\omega, t) & =\frac{\Theta_{j}(\omega, t)+\Theta_{j}(\omega, 2 \pi-t)}{2}, \\
W_{r}^{\prime}(\omega, t) & =\left\{\sum_{j=1}^{\infty} \sup _{N \in I_{j}}\left|\frac{S_{N}\left(\mathscr{X}_{f}^{\prime}(\omega, t)\right)}{\tau_{N}}-\Theta_{j}^{\prime}(\omega, t)\right|^{r}\right\}^{1 / r} .
\end{aligned}
$$


Since $W_{r}^{\prime}(\omega, t) \leqslant\left(W_{r}(\omega, t)+W_{r}(\omega, 2 \pi-t)\right) / 2$, we have $\left\|W_{r}^{\prime}\right\|_{G, \nu} \leqslant C_{r} \Delta_{r}$. Thus, there exists an $\omega \in \Omega$ such that

$$
\int_{X} \exp \left(\left[\frac{W_{r}^{\prime}(\omega, t)}{C_{r} \Delta_{r}}\right]^{2}\right) d t \leqslant 4
$$

And so, by means of Jensen's inequality:

$$
\left\|W_{r}^{\prime}(\omega, \cdot)\right\|_{G, m} \leqslant C_{r} \Delta_{r}
$$

Put for any positive integers $k, j$, and $t \in X$ :

$$
\begin{gathered}
\beta_{k}^{*}=\beta_{k} g_{k}(\omega), \quad f^{*}(t)=\sum_{k=1}^{\infty} \beta_{k}^{*} \cos 2 \pi l_{k} t, \quad f_{j}^{*}=\sum_{k>j} \beta_{k}^{*} \cos 2 \pi l_{k} t \\
W_{r}\left(f^{*}\right)=\left\{\sum_{j=1}^{\infty} \sup _{N \in I_{j}}\left|\frac{S_{N}\left(f^{*}\right)}{N\left\|f_{j}^{*}\right\|_{2}}-\frac{f_{j}^{*}}{\left\|f_{j}^{*}\right\|_{2}}\right|^{r}\right\}^{1 / r} .
\end{gathered}
$$

Let $\eta=\left(\eta_{n}\right)_{n \geqslant 1}$ be some sequence of reals decreasing to 0 and such that

$$
\sum_{j=1}^{\infty} \frac{1}{b_{j}}\left(\sum_{k<j} \beta_{k} \eta_{k}\right)^{r} \leqslant \Delta_{r}^{r}
$$

We choose here a sequence $\mathscr{L}$ satisfying (2.2) and (2.3). According to the observation made right after definition of $\mathscr{L}$ in Section 2, this is always possible. We use Berkes' result (cf. [1] and proof of the sufficiency part on p. 59). Let $0<\eta<1$ be arbitrary but fixed. There exists a suitable enlarging $\left(X^{\prime}, m^{\prime}\right)$ of $(X, m)$ and a sequence $\left(Y_{k}\right)_{k \geqslant 1}$ of i.i.d. random variables defined on $\left(X^{\prime}, m^{\prime}\right)$ such that

$$
\forall k \geqslant 1, \quad m\left\{t \in X:\left|\cos 2 \pi l_{k} t-Y_{k}(t)\right|>\frac{l_{k}}{l_{k+1}}\right\} \leqslant \frac{l_{k}}{l_{k+1}} .
$$

We denote $A=\left\{t \in X: \forall k \geqslant 1,\left|\cos 2 \pi l_{k} t-Y_{k}(t)\right| \leqslant l_{k} / l_{k+1}\right\}$. Since $m(A) \geqslant 1-\eta$ and for $t \in A$ and positive integers $N<M$

$$
\left|\sum_{k=N}^{M} \beta_{k} Y_{k}(t)-\sum_{k=N}^{M} \beta_{k} \cos 2 \pi l_{k} t\right| \leqslant \sum_{k=N}^{M} \eta_{k} \beta_{k} \longrightarrow 0 \quad(N, M \rightarrow \infty),
$$

we deduce from Sidon's theorem for lacunary trigonometric series that

$$
m^{\prime}\left\{t \in X^{\prime}: \sum_{k} \beta_{k} Y_{k}(t) \text { converges }\right\} \geqslant 1-\eta .
$$

By invoking now the three series theorem, it follows that the sequence $\mathscr{Y}$ is an i.i.d. sequence of centered square integrable random variables. Put 
next for all positive integers $j: R_{j}(t)=\sum_{k>j} \beta_{k}^{*} Y_{k}(t)$. Then $\left\|R_{j}\right\|_{2}=\left\|f_{j}^{*}\right\|_{2}$, $m(A) \geqslant 1-\eta$ and for any $t \in A$

$$
\sum_{j=1}^{\infty}\left|\Theta_{j}^{*}(t)-R_{j}(t)\right|^{r} \leqslant \sum_{j=1}^{\infty} \frac{1}{b_{j}}\left(\sum_{k<j} \beta_{k} \eta_{k}\right)^{r} \leqslant \Delta_{r}^{r}
$$

by virtue of the assumption $(h)$ made. Indeed, it suffices to choose a sequence $\eta$ depending on $\beta$ decreasing sufficiently fast to 0 . Therefore,

$$
\left(\sum_{j=1}^{\infty}\left|\Theta_{j}^{*}(t)-R_{j}(t)\right|^{r}\right)^{1 / r} \leqslant \Delta_{r} \quad(\forall t \in A) .
$$

Put finally

$$
W_{r}^{*}=\left\{\sum_{j=1}^{\infty} \sup _{N \in I_{j}}\left|\frac{S_{N}\left(f^{*}\right)}{N\left\|f_{j}^{*}\right\|_{2}}-\frac{R_{j}}{\left\|f_{j}^{*}\right\|_{2}}\right|^{r}\right\}^{1 / r} .
$$

Using now triangle inequality together with the above estimates gives: $\mathbf{1}_{A} W_{r}^{*} \leqslant C_{r} \Delta_{r}+W_{r}\left(f^{*}\right)$. Hence

$$
\left\|1_{A} W_{r}^{*}\right\|_{G, m} \leqslant C_{r} \Delta_{r}
$$

We can now state the following theorem.

Theorem 4.1 (First uniform approximation theorem). Let $\theta \in] 0,1[$ be irrational and denote by $\tau$ the rotation on the circle $X=[0,1[=\mathbf{R} / \mathbf{Z}$, $m$ being the normalized Lebesgue measure on $X$. Let also $0<\eta<1$ be arbitrary but fixed.

There exist $h \in L^{2}(m)$ and a measurable subset $A$ of $X$ with $m(A) \geqslant$ $1-\eta$, as well as a sequence $\mathscr{Y}=\left(Y_{j}\right)_{j \geqslant 1}$ of i.i.d. centered square integrable random variables defined on a suitable enlarging $\left(X^{\prime}, m^{\prime}\right)$ of $(X, m)$ and two sequences of positive reals $\left(N_{j}\right)_{j \geqslant 1},\left(\mu_{N}\right)_{N \geqslant 1}$ with $\left(N_{j}\right)_{j \geqslant 1}$ increasing to infinity and $\left(\mu_{N}\right)_{N \geqslant 1}$ converging to 1 such that if

$$
W_{r}(h, \mathscr{Y})=\left\{\sum_{j=1}^{\infty} \sup _{N_{j} \leqslant N<N_{j+1}}\left|\frac{S_{N} h}{\left\|S_{N} h\right\|_{2}}-\mu_{N} \frac{\sum_{k>j}\left\langle h, e_{k}\right\rangle Y_{k}}{\left(\sum_{k>j}\left\langle h, e_{k}\right\rangle^{2}\right)^{1 / 2}}\right|^{r}\right\}^{1 / r},
$$

then for some $r>2$,

$$
\left\|\mathbf{1}_{A} W_{r}(h, \mathscr{Y})\right\|_{G, m}<\infty
$$

The next result singles out some extremal behavior of CLT-normalized partial sums. We indeed give an example in $L^{2}(m)$ such that the set of CLT-normalized partial sums is nearly close to some orthonormal system. 
Theorem 4.2 (Second uniform approximation theorem). Let $\theta \in] 0,1[$ be irrational and denote by $\tau$ the rotation on the circle $X$. Let also $0<\rho<1$ be arbitrary but fixed.

There exist $h \in L^{2}(m)$ as well as an orthonormal system of functions $\Psi=\left(\Psi_{j}\right)_{j \geqslant 1}$ defined on a suitable enlarging $\left(X^{\prime}, m^{\prime}\right)$ of $(X, m)$ and two sequences of positive reals $\left(N_{j}\right)_{j \geqslant 1},\left(\mu_{N}\right)_{N \geqslant 1}$ with $\left(N_{j}\right)_{j \geqslant 1}$ increasing to infinity and lim $\sup _{N \rightarrow \infty}\left|\mu_{N}-1\right| \leqslant \rho$ such that

$$
m\left\{t: \limsup _{j \rightarrow \infty} \sup _{N_{j} \leqslant N<N_{j+1}}\left|\frac{S_{N} h(t)}{\left\|S_{N} h\right\|_{2, m}}-\mu_{N} \Psi_{j}(t)\right| \leqslant \rho\right\}=1 .
$$

P r o o f. Let $0<\rho<1$ be fixed and choose $\beta_{k}=(1+\rho)^{-k}, \varepsilon_{k}=$ $(1+\rho / 2)^{-k}(k \geqslant 1)$. We may already notice that the following quantity appearing in (3.6):

$$
\sum_{j=1}^{\infty}\left[\frac{\varepsilon_{j}}{b_{j}} \sqrt{\ln \sharp\left(I_{j}\right)}\right]^{r}
$$

is finite for any positive $r$. To prove the theorem we shall combine three results: a part of the proof of Proposition 3.1, a criterion for quasiorthogonality, and finally Schur's theorem (see, for instance, $[16$, p. 56]).

Once again we assume both sequences $g$ and $g^{\prime}$ to be independent Rademacher sequences. It will be necessary to separately examinate the perturbating term $s_{N}$ defined in (2.6). It is why we put $S_{N}=S_{N}^{0}+s_{N}$. According to the decomposition (2.7), we have for $N \in I_{j}=\left[N_{j}, N_{j+1}[\right.$ :

$$
\frac{S_{N}}{\tau_{N}}-\Theta_{j}=Y_{N}+Z_{N}+U_{N}=\frac{S_{N}^{0}}{\tau_{N}}+U_{N} .
$$

Fix some $r>2$. From the proof of Proposition 3.1, we have by (3.5) and (3.6)

$$
\|\mathscr{Y}\|_{G, \nu}+\|\mathscr{Z}\|_{G, \nu} \leqslant C_{r} .
$$

By Minkowski's inequality, putting

$$
V_{r}(\mathscr{X})=V_{r}=\left\{\sum_{j=1}^{\infty} \sup _{N \in I_{j}}\left|\frac{S_{N}^{0}}{\tau_{N}}\right|^{r}\right\}^{1 / r}
$$

we obtain $\left\|V_{r}\right\|_{G, \nu} \leqslant C_{r}$. We use the notation employed at the beginning of the section and denote $V_{r}^{\prime}=V_{r}(\mathscr{X})$. Similarly we can then infer that $\left\|V_{r}^{\prime}\right\|_{G, \nu} \leqslant C_{r}$. In other words, $\mathbf{E} \int_{X} \exp \left[\left(V_{r}^{\prime}\right)^{2} / C_{r}\right] d t \leqslant 2$. This implies that for any $p \geqslant 1$ we have $\left\|\left(V_{r}^{\prime}\right)^{r}\right\|_{2 p / r, \nu} \leqslant C_{p, r}$, where $C_{p, r}$ depends on $p, r$ only. Choose $p=\lfloor r / 2\rfloor+1$. By monotonicity of the $\|\cdot\|_{p}$ norms, we have $\left\|\left(V_{r}^{\prime}\right)^{r}\right\|_{1, \nu} \leqslant C_{r}$. This implies that there exists an $\omega \in \Omega$ such that

$$
\int_{X} \sum_{j=1}^{\infty} \sup _{N \in I_{j}}\left|\frac{S_{N}^{0}(\omega, t)}{\tau_{N}}\right|^{r} d t \leqslant C_{r}^{\prime}
$$


We can take $C_{r}^{\prime}=2 C_{r}$. With the notation used in (4.3), we have

$$
\int_{X} \sum_{j=1}^{\infty} \sup _{N \in I_{j}}\left|\frac{S_{N}^{0}\left(f^{*}\right)(t)}{\tau_{N}}\right|^{r} d t \leqslant C_{r}^{\prime} .
$$

Now since $S_{N} / \tau_{N}-f_{j}^{*} /\left\|f_{j}^{*}\right\|_{2}=S_{N}^{0}\left(f^{*}\right) / \tau_{N}+s_{N}\left(f^{*}\right) / \tau_{N},\left\|s_{N}\left(f^{*}\right) / \tau_{N}\right\|_{\infty} \leqslant \rho$ and $b_{j}=(1+\rho)^{-j} / \rho$, from the last obtained estimate we deduce

$$
m\left\{t: \limsup _{j \rightarrow \infty} \sup _{N \in I_{j}}\left|\frac{S_{N}\left(f^{*}\right)(t)}{\tau_{N}}-\frac{f_{j}^{*}(t)}{\left\|f_{j}^{*}\right\|_{2}}\right| \leqslant \rho\right\}=1 .
$$

But for $j<k$

$$
\left\langle\frac{f_{j}^{*}}{\left\|f_{j}^{*}\right\|_{2}}, \frac{f_{k}^{*}}{\left\|f_{k}^{*}\right\|_{2}}\right\rangle=(1+\rho)^{-(k-j)}
$$

Then according to the criterion 7.4 .3 in $\left[21\right.$, p. 139], the system $\left(f_{j}^{*} /\left\|f_{j}^{*}\right\|_{2}\right.$, $j \geqslant 1$ ) is a quasi-orthogonal system. By virtue of Schur's theorem, there exists an orthonormal system $\Psi=\left(\Psi_{j}, j \geqslant 1\right)$ defined on a suitable enlarging of $(X, m)$ extending the system $\left(f_{j}^{*} /\left\|f_{j}^{*}\right\|_{2}, j \geqslant 1\right)$. We achieve the proof by putting $\mu_{N}=\tau_{N} /\left\|S_{N}\left(f^{*}\right)\right\|_{2}$ for $j \geqslant 1$ and $N \in I_{j}$.

5. CLT. In view of proving a CLT for $\mathscr{X}$ defined in (2.4), we slightly modify the definition on $f$. First we assume that $\left(l_{k}\right)$ is $r$-lacunary with $r \geqslant 3$. Let $\beta^{1}=\left(\beta_{k}^{1}\right)_{k \geqslant 1}, \beta^{2}=\left(\beta_{k}^{2}\right)_{k \geqslant 1}, \beta^{1}, \beta^{2} \in \ell^{2}$, and put $\rho=\left(\rho_{k}\right)_{k \geqslant 1}$ with $\rho_{k}=\beta_{k}^{1}+i \beta_{k}^{2}$ and

$$
F(t)=\sum_{k=1}^{\infty} \operatorname{Re}\left(\rho_{k} \overline{e_{l_{k}}(t)}\right) \quad(\forall t \in X) .
$$

We give simple conditions ensuring that the property $F \in$ CLT holds true. We use the decomposition of partial sums made in Section 2 and the SalemZygmund method, which we detail for the convenience of the reader. Next we show by simple randomization and standard considerations how this can be used to prove that $\mathscr{X}(\omega) \in$ CLT for almost all $\omega$.

Theorem 5.1. Assume that the following condition is satisfied:

$$
\lim _{j \rightarrow \infty} \frac{\varepsilon_{j}+\left|\rho_{j}\right|}{\left(\sum_{k>j}\left|\rho_{k}\right|^{2}\right)^{1 / 2}}=0 .
$$

Then $F \in$ CLT.

Note that the conclusion of the theorem holds true independently of the choice of the signs of $\beta_{k}^{s}, s=1,2$, and that the set of functions $F$ such that (5.2) hold is a subspace of $L^{2}(m)$. We give a proof with detailed computations, without using symbols $o$, $O$, for the convenience of the reader and for later use. But the reader may also directly switch to the SalemZygmund result ([18, assertion (v), p. 337]) for rests of trigonometric series, 
once having passed the reduction step at the paragraph including (5.5). Some classical estimates for products of complex exponentials collected in the following technical lemma are necessary.

Lemma 5.1. (a) Let $\left\{z_{k}, k \in \Delta\right\}$ be complex numbers satisfying

$$
h_{0}=\sum_{k \in \Delta} e^{-z_{k}^{2} / 2}\left|\left[\frac{1}{4} e^{\left|z_{k}\right|^{2} / 2}\left|z_{k}\right|^{4}+\frac{1}{3} \frac{\left|z_{k}\right|^{3}}{\left|1+z_{k}\right|} e^{\left|z_{k}\right|}\right]\right| \leqslant \frac{1}{2} .
$$

Then

$$
\left|\frac{\prod_{k \in \Delta} e^{z_{k}}}{\prod_{k \in \Delta}\left(1+z_{k}\right) e^{z_{k}^{2} / 2}}-1\right| \leqslant 2 h_{0} .
$$

(b) Assume that $z_{k}$ are purely imaginary, and that

$$
h=\sum_{k \in \Delta}\left[\frac{\left|z_{k}\right|^{4}}{4} e^{\left|z_{k}\right|^{2}}+\frac{\left|z_{k}\right|^{3}}{3 \sqrt{1+\left|z_{k}\right|^{2}}} e^{\left|z_{k}\right|+\left|z_{k}\right|^{2} / 2}\right] \leqslant \frac{1}{2} ;
$$

then

$$
\left|\prod_{k \in \Delta} e^{z_{k}}-\prod_{k \in \Delta}\left(1+z_{k}\right) e^{z_{k}^{2} / 2}\right| \leqslant 2 h .
$$

P r o o f. Put $a_{k}=e^{z_{k}} /\left(\left(1+z_{k}\right) e^{z_{k}^{2} / 2}\right)-1, k \in \Delta$. Using the following inequality valid for any complex number $z$ : $\left|e^{z}-(1+z) e^{z^{2} / 2}\right| \leqslant \frac{1}{4} \mid 1+$ $\left.z|| z\right|^{4} e^{|z|^{2} / 2}+\frac{1}{3}|z|^{3} e^{|z|}$, we get

$$
\left|a_{k}\right| \leqslant\left|e^{-z_{k}^{2} / 2}\right|\left[\frac{1}{4} e^{\left|z_{k}\right|^{2} / 2}\left|z_{k}\right|^{4}+\frac{1}{3} \frac{\left|z_{k}\right|^{3}}{\left|1+z_{k}\right|} e^{\left|z_{k}\right|}\right] .
$$

Thus $\sum_{k \in \Delta}\left|a_{k}\right|=h \leqslant \frac{1}{2}$, which by means of inequality 3.8 .8 in $[15$, p. 314] implies

$$
\left|\frac{\prod_{k \in \Delta} e^{z_{k}}}{\prod_{k \in \Delta}\left(1+z_{k}\right) e^{z_{k}^{2} / 2}}-1-\sum_{k \in \Delta} a_{k}\right| \leqslant \frac{h^{2}}{1-h} .
$$

Hence (a) follows. Concerning (b), observe that $\left|1+z_{k}\right|\left|e^{z_{k}^{2} / 2}\right|=$ $|1+i| z_{k}|| e^{-\left|z_{k}\right|^{2} / 2} \leqslant 1$, hence $\left|\prod_{k \in \Delta}\left(1+z_{k}\right) e^{z_{k}^{2} / 2}\right| \leqslant 1$.

Multiplying both sides of inequality in (a) by $\prod_{k \in \Delta}\left(1+z_{k}\right) e^{z_{k}^{2} / 2}$ gives

$$
\begin{aligned}
\left|\prod_{k \in \Delta} e^{z_{k}}-\prod_{k \in \Delta}\left(1+z_{k}\right) e^{z_{k}^{2} / 2}\right| & \leqslant 2 \sum_{k \in \Delta}\left[\frac{1}{4}\left|z_{k}\right|^{4} e^{\left|z_{k}\right|^{2}}+\frac{1}{3} \frac{\left|z_{k}\right|^{3}}{\sqrt{1+\left|z_{k}\right|^{2}}} e^{\left|z_{k}\right|+\left|z_{k}\right|^{2} / 2}\right] \\
& =2 h .
\end{aligned}
$$

Pr o of of $\mathrm{T}$ h e or e $\mathrm{m}$ 5.1. We observe that

$$
S_{N} F(t)=N \sum_{k=1}^{\infty} \operatorname{Re}\left[\rho_{k} \overline{V_{N}\left(l_{k} \theta\right) e_{l_{k}}(t)}\right] .
$$


Introduce some notation; put for any $j \geqslant 1, N \in I_{j}$, and $t \in X$ :

$$
\begin{gathered}
\check{S}_{N} F(t)=N \sum_{k>j} \operatorname{Re}\left[\rho_{k} \overline{V_{N}\left(l_{k} \theta\right) e_{l_{k}}(t)}\right], \\
\widehat{S}_{N} f(t)=N \sum_{k \leqslant j} \rho_{k} \operatorname{Re}\left[\rho_{k} \overline{V_{N}\left(l_{k} \theta\right) e_{l_{k}}(t)}\right], \\
\check{S}_{N} F(t)=S_{N}^{\prime} F+s_{N} F, \quad S_{N}^{\prime} F=N \sum_{k<j} \operatorname{Re}\left[\rho_{k} \overline{V_{N}\left(l_{k} \theta\right) e_{l_{k}}(t)}\right], \\
F_{j}(t)=\sum_{k>j} \operatorname{Re}\left[\rho_{k} \overline{e_{l_{k}}(t)}\right], \quad c_{j}=\left(\sum_{k>j}\left|\rho_{k}\right|^{2}\right)^{1 / 2}, \quad \sigma_{N}=N c_{j} .
\end{gathered}
$$

Then, similarly to what was made in Section 2,

$$
\begin{gathered}
\left\|\check{S}_{N} f-N F_{j}\right\|^{2}=N^{2} \sum_{k>j}\left|\rho_{k}\right|^{2}\left|V_{N}\left(l_{k} \theta\right)-1\right|^{2} \leqslant 4 N^{2} \varepsilon_{j+1}^{2} c_{j}^{2}, \\
\left\|S_{N}^{\prime}\right\|^{2} \leqslant 4 N^{2} c_{0}^{2} \varepsilon_{j}^{2}, \quad\left\|s_{N}\right\| \leqslant N\left|\rho_{j}\right| .
\end{gathered}
$$

Thus,

$$
\left\|\frac{S_{N} F}{\sigma_{N}}-\frac{F_{j}}{c_{j}}\right\| \leqslant\left\|\frac{\check{S}_{N} F}{\sigma_{N}}-\frac{F_{j}}{c_{j}}\right\|+\left\|\frac{\widehat{S}_{N} F}{\sigma_{N}}\right\| \leqslant 2 \varepsilon_{j+1}+\frac{2 \varepsilon_{j}+\left|\rho_{j}\right|}{c_{j}} \longrightarrow 0
$$

as $N$ tends to infinity under the assumptions made. And by triangle inequality:

$$
\lim _{N \rightarrow \infty} \frac{\left\|S_{N} F\right\|_{2}}{\sigma_{N}}=1 .
$$

Using now the elementary inequality $\left|e^{i u}-e^{i v}\right| \leqslant 2|\sin ((v-u) / 2)| \leqslant|v-u|$ gives

$$
\begin{aligned}
& \left|\int_{0}^{1}\left(\exp \left(i \lambda \frac{S_{N} F(t)}{\sigma_{N}}\right)-\exp \left(i \lambda \frac{F_{j}(t)}{c_{j}}\right)\right) d t\right| \leqslant|\lambda| \mid \frac{S_{N} F}{\sigma_{N}}-\frac{F_{j}}{c_{j}} \|_{1} \\
& \quad \leqslant|\lambda|\left\{2 \varepsilon_{j+1}+\frac{2 \varepsilon_{j}+\left|\rho_{j}\right|}{c_{j}}\right\} \longrightarrow 0
\end{aligned}
$$

as $N$ tends to infinity. Let now $\kappa: \mathbf{N} \rightarrow \mathbf{N}$ be some increasing map satisfying $\sum_{k>\kappa(j)}\left|\rho_{k}\right|^{2} / c_{j}^{2} \leqslant \varepsilon_{j}^{2}, j \geqslant 1$, and put $\Delta_{j}=\left[j, \kappa(j)\left[, F_{j}^{\prime}=\right.\right.$ $\sum_{j<k \leqslant \kappa(j)} \operatorname{Re}\left[\rho_{k} \overline{e_{l_{k}}(t)}\right], j \geqslant 1$. Again,

$$
\left|\int_{0}^{1}\left(\exp \left(i \lambda \frac{F_{j}(t)}{b_{j}}\right)-\exp \left(i \lambda \frac{F_{j}^{\prime}(t)}{b_{j}}\right)\right) d t\right| \leqslant|\lambda| \frac{\left\|F_{j}-F_{j}^{\prime}\right\|_{1}}{c_{j}} \leqslant|\lambda| \varepsilon_{j} \longrightarrow 0
$$

as $j \rightarrow \infty$. Put now for $k>j$ and $t \in X: z_{k}^{j}(t)=\operatorname{Re}\left[\rho_{k} \overline{e_{l_{k}}(t)}\right] / c_{j}$. Then

$$
\sup _{k>j} \sup _{t \in X}\left|z_{k}^{j}(t)\right| \leqslant \sup _{k>j} \frac{\left|\rho_{k}\right|}{c_{k}} \longrightarrow 0
$$


as $j$ tends to infinity by assumption. Let also $\Lambda \in \mathbf{N}$. Then for some finite integer $J=J(\Lambda)$ depending on $\Lambda$ only, we have $\sup _{k>j} \sup _{t \in X}\left|z_{k}^{j}(t)\right| \leqslant \frac{1}{2}$, for any $j \geqslant J$. The quantity $h$ appearing in Lemma 5.1 can now be estimated as follows:

$$
\begin{aligned}
h & \leqslant \Lambda^{3}\left[\frac{1}{8} e^{1 / 4}+\frac{\sqrt{2}}{3} e^{1 / 2+1 / 8}\right] \sum_{k \in \Delta_{j}}\left|z_{k}^{j}(t)\right|^{3} \\
& \leqslant C \Lambda^{3} \frac{\sum_{k>j}\left|\rho_{k}\right|^{3}}{\left(\sum_{k>j}\left|\rho_{k}\right|^{2}\right)^{3 / 2}} \leqslant C \Lambda^{3} \sup _{k>j} \frac{\left|\rho_{k}\right|}{c_{k}}
\end{aligned}
$$

which tends to 0 as $j$ tends to infinity. Redefining $J$ if necessary, we deduce: for all $t \in X$ and $j \geqslant J$,

$$
\left|\exp \left(i \lambda \frac{F_{j}^{\prime}(t)}{b_{j}}\right)-\prod_{k \in \Delta_{j}} z_{k}^{j}(t) \exp \left(\frac{z_{k}^{j}(t)^{2}}{2}\right)\right| \leqslant C \Lambda^{3} \sup _{k>j} \frac{\left|\rho_{k}\right|}{c_{k}} .
$$

Integrating on $X$ leads for $j \geqslant J$ to

$$
\left|\int_{0}^{1}\left(\exp \left(i \lambda \frac{F_{j}^{\prime}(t)}{b_{j}}\right)-\prod_{k \in \Delta_{j}} z_{k}^{j}(t) \exp \left(\frac{z_{k}^{j}(t)^{2}}{2}\right)\right) d t\right| \leqslant C \Lambda^{3} \sup _{k>j} \frac{\left|\rho_{k}\right|}{c_{k}} .
$$

Put now for any integer $j \geqslant 1$ :

$$
\prod_{j}(\lambda, t)=\prod_{k \in \Delta_{j}}\left(1+i \lambda z_{k}^{j}(t)\right), \quad B_{j}(t)=\sum_{k \in \Delta_{j}}\left(z_{k}^{j}(t)\right)^{2} .
$$

We have $\int_{0}^{1} \prod_{j}(\lambda, t) d t=1$. Indeed the product being $\prod_{k}\left(1+i \lambda\left[\beta_{k}^{1} \cos 2 \pi l_{k} t+\right.\right.$ $\left.\left.\beta_{k}^{2} \sin 2 \pi l_{k} t\right]\right)$ is representable as a sum of 1 plus a linear combination of $\cos 2 \pi\left(l_{k_{1}} \pm \cdots \pm l_{k_{r}}\right)$ or $\sin 2 \pi\left(l_{k_{1}} \pm \cdots \pm l_{k_{r}}\right)$. Since we assumed $\left(l_{k}\right)$ to be $r$-lacunary with $r \geqslant 3$, the fact that the representation of a number $n$ as $n=l_{k_{1}} \pm \cdots \pm l_{k_{r}}$ is unique allows one to conclude. We can thus factorize as follows:

$$
\begin{aligned}
& \left|\int_{0}^{1} \prod_{j}(\lambda, t) \cdot \exp \left(-\frac{\lambda^{2} B_{j}(t)}{2}\right) d t-\exp \left(-\frac{\lambda^{2}}{4}\right)\right| \\
& \quad=\left|\int_{0}^{1} \prod_{j}(\lambda, t)\left[\exp \left(-\frac{\lambda^{2} B_{j}(t)}{2}\right)-\exp \left(-\frac{\lambda^{2}}{4}\right)\right] d t\right| \\
& \quad \leqslant \int_{0}^{1}\left|\prod_{j}(\lambda, t)\right|\left|\exp \left(-\frac{\lambda^{2} B_{j}(t)}{2}\right)-\exp \left(-\frac{\lambda^{2}}{4}\right)\right| d t .
\end{aligned}
$$

Now

$$
\left|\prod_{j}(\lambda, t)\right|=\prod_{j<k \leqslant \kappa(j)}\left(1+|\lambda|^{2}\left|z_{k}^{j}(t)\right|^{2}\right)^{1 / 2} \leqslant \exp \left(\frac{\lambda^{2} B_{j}(t)}{2}\right) .
$$


Consequently,

$$
\begin{aligned}
& \int_{0}^{1}\left|\prod_{j}(\lambda, t)\right| \cdot\left|\exp \left(-\frac{\lambda^{2} B_{j}(t)}{2}\right)-\exp \left(-\frac{\lambda^{2}}{4}\right)\right| d t \\
& \leqslant \int_{0}^{1}\left|1-\exp \left(-\frac{\lambda^{2}}{2}\left[\frac{1}{2}-B_{j}(t)\right]\right)\right| d t .
\end{aligned}
$$

But

$$
\begin{aligned}
B_{j}(t)=\frac{1}{c_{j}^{2}}\left\{\sum_{k \in \Delta_{j}} \frac{\left(\beta_{k}^{1}\right)^{2}+\left(\beta_{k}^{2}\right)^{2}}{2}-\sum_{k \in \Delta_{j}} \frac{\left(\beta_{k}^{1}\right)^{2}-\left(\beta_{k}^{2}\right)^{2}}{2} \cos 4 \pi l_{k} t\right. \\
\left.+\sum_{k \in \Delta_{j}} \beta_{k}^{1} \beta_{k}^{2} \sin 4 \pi l_{k} t\right\} .
\end{aligned}
$$

Put $C_{j}(t)=\frac{1}{2}-B_{j}(t)-c_{j}^{-2} \sum_{k>\kappa_{j}}\left(\left(\beta_{k}^{1}\right)^{2}+\left(\beta_{k}^{2}\right)^{2}\right) / 2$. Then since $0 \leqslant B_{j}(t) \leqslant 2$,

$$
\begin{aligned}
& \int_{0}^{1}\left|1-\exp \left(-\frac{\lambda^{2}}{2}\left[\frac{1}{2}-B_{j}(t)\right]\right)\right| d t \\
& \quad \leqslant \frac{\Lambda^{2}}{2} e^{3 \Lambda^{2} / 4}\left[\frac{1}{c_{j}^{2}} \sum_{k>\kappa_{j}} \frac{\left(\beta_{k}^{1}\right)^{2}+\left(\beta_{k}^{2}\right)^{2}}{2}+\left\|C_{j}\right\|_{2}\right] .
\end{aligned}
$$

It remains to observe that

$$
\left\|C_{j}\right\|_{2}^{2} \leqslant \frac{1}{4} \sum_{k \in \Delta_{j}} \frac{\left|\rho_{k}\right|^{4}}{c_{j}^{4}} \leqslant \frac{1}{4} \sup _{k>j} \frac{\left|\rho_{k}\right|^{2}}{c_{k}^{2}} \longrightarrow 0
$$

as $j$ tends to infinity by assumption. Collecting these various estimates finally gives: for $|\lambda| \leqslant \Lambda$ and $j \geqslant J=J(\Lambda), N \in I_{j}$

$$
\begin{aligned}
& \left|\int_{0}^{1} \exp \left(i \lambda \frac{S_{N} F(t)}{\sigma_{N}}\right) d t-\exp \left(-\frac{\lambda^{2}}{4}\right)\right| \leqslant \Lambda\left\{2 \varepsilon_{j+1}+\frac{2 \varepsilon_{j}+\left|\rho_{j}\right|}{c_{j}}\right\}+\varepsilon_{j} \Lambda \\
& +C \Lambda^{3} \sup _{k>j} \frac{\left|\rho_{k}\right|}{c_{k}}+C \Lambda^{2} \exp \left(\frac{3}{4} \Lambda^{2}\right) \sup _{k>j} \frac{\left|\rho_{k}\right|^{2}}{c_{k}^{2}} \longrightarrow 0
\end{aligned}
$$

as $N$ tends to infinity. This and property (5.3) finally achieve the proof.

Let us now consider the random Fourier series defined in (2.4) and assume that the sequences $g, g^{\prime}$ are independent and isonormal.

Theorem 5.2 (CLT for sample paths). Assume that

$$
\lim _{j \rightarrow \infty} \frac{\left|\beta_{j}\right| \ln j}{b_{j}}=0, \quad \sum_{j \geqslant 1} \frac{\varepsilon_{j}^{2} \ln N_{j+1}}{b_{j}^{2}}<\infty .
$$

Then almost all sample paths of $\mathscr{X}$ satisfy the CLT. 
Pr o o f. We may write

$$
\begin{gathered}
\mathscr{X}(\omega, t)=\sum_{k \in \mathbf{N}} \operatorname{Re}\left(\rho_{k}(\omega) \overline{e_{l_{k}}}(t)\right) \\
\text { with } \quad \rho_{k}(\omega)=\beta_{k} g_{k}(\omega)+i \beta_{k} g_{k}^{\prime}(\omega) \quad(k \geqslant 1) .
\end{gathered}
$$

Put

$$
\varphi_{N}(\omega)^{2}=\sum_{k>j}\left(g_{k}(\omega)^{2}+g_{k}^{\prime}(\omega)^{2}\right) \beta_{k}^{2} \quad\left(N \in I_{j}, j \geqslant 1\right) .
$$

Consider the Gaussian chaos of order 2

$$
B_{j}=\sum_{k>j}\left(\frac{\beta_{k}}{b_{j}}\right)^{2}\left[g_{k}^{2}+\frac{g_{k}^{\prime 2}-2}{2}\right] .
$$

By the hypercontractivity properties of Gaussian chaos (see, for instance, $[14$, inequality 3.8, p. 65$]$ ), for any integer $q \geqslant 2$,

$$
\left\|B_{j}\right\|_{q, \mathbf{P}} \leqslant q\left\|B_{j}\right\|_{2, \mathbf{P}}
$$

Let $w_{j}=\left\|B_{j}\right\|_{2, \mathbf{P}}$ and $\alpha_{j}=1 /\left(2 e w_{j}\right)$. Then

$$
\mathbf{E} \exp \left(\alpha_{j}\left|B_{j}\right|\right) \leqslant 1+\alpha_{j}\left\|B_{j}\right\|_{1, \mathbf{P}}+\sum_{q=2}^{\infty} \frac{1}{q !}\left(\alpha_{j} q\right)^{q} w_{j}^{q} .
$$

Using (see, for instance, $[15$, p. 183 , the sixth paragraph]) the elementary estimate $n !>\sqrt{2 \pi n} n^{n} e^{-n} \exp \left(12 n+\frac{1}{4}\right)^{-1}$ valid for any positive integer $n$, we deduce

$$
\sum_{q=2}^{\infty} \frac{1}{q !}\left(\alpha_{j} w_{j} q\right)^{q} \leqslant \frac{1}{2 \sqrt{\pi}} \sum_{q=2}^{\infty} 2^{-q}=\frac{1}{4 \sqrt{\pi}} .
$$

Thus $\mathbf{E} \exp \left(\alpha_{j}\left|B_{j}\right|\right) \leqslant C$ (with $C=1+1 /(2 e)+1 /(4 \sqrt{\pi})$ ). Applying then Chebyshev inequality gives for any positive real $\eta$

$$
\mathbf{P}\left\{\left|B_{j}\right| \geqslant \eta\right\} \leqslant C \exp \left(-\frac{\eta}{2 e w_{j}}\right) .
$$

Now $\left\|B_{j}\right\|_{2, \mathbf{P}}^{2}=2\left(\mathbf{E} N(0,1)^{4}-1\right) \sum_{k>j} \beta_{k}^{4} / b_{j}^{4}$. By assumption,

$$
\sum_{k>j} \frac{\beta_{k}^{4}}{b_{j}^{4}}=o\left(\sum_{k>j} \frac{\beta_{k}^{2}}{b_{j}^{2} \ln ^{2} j}\right)=o\left(\frac{1}{\ln ^{2} j}\right) .
$$

Thus,

$$
w_{j}=o\left(\frac{1}{\ln j}\right) .
$$


Let $\rho>0$ be such that $2 e \rho<\eta$. For $j$ large, $w_{j} \leqslant \rho / \ln j$, and so

$$
\mathbf{P}\left\{\left|B_{j}\right| \geqslant \eta\right\} \leqslant C \exp \left(-\frac{\eta}{2 e w_{j}}\right) \leqslant \exp \left(-\frac{\eta}{2 e \rho} \ln j\right) .
$$

By the Borel-Cantelli lemma and using the fact that $\eta$ can be arbitrarily small, we deduce $\left|B_{j}\right| \stackrel{\text { a.s. }}{=} o(1)$, or

$$
\lim _{j \rightarrow \infty} \frac{1}{b_{j}^{2}} \sum_{k>j}\left(g_{k}^{2}+\left(g_{k}^{\prime}\right)^{2}\right) \beta_{k}^{2} \stackrel{\text { a.s. }}{=} 2 .
$$

Therefore,

$$
\lim _{N \rightarrow \infty} \frac{N \varphi_{N}(\omega)}{\tau_{N}}=\lim _{j \rightarrow \infty} \frac{1}{b_{j}^{2}} \sum_{k>j}\left(g_{k}^{2}(\omega)+\left(g_{k}^{\prime}(\omega)\right)^{2}\right) \beta_{k}^{2}=2 \quad \text { P-a.s. }
$$

The assumption of Theorem 5.1 here reduces to

$$
\lim _{j \rightarrow \infty} \frac{\varepsilon_{j}+\left(g_{j}^{2}(\omega)+\left(g_{j}^{\prime}(\omega)\right)^{2}\right)^{1 / 2} \mid \beta_{j} !}{b_{j}}=0,
$$

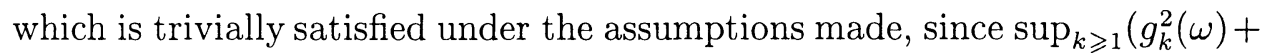
$\left(g_{k}^{\prime}(\omega)\right)^{2} / \ln k<\infty, \mathbf{P}$-a.s. Applying now Theorem 5.1 gives for $\mathbf{P}$-a.a. trajectories of $\mathscr{X}(\omega)$ :

$$
\frac{S_{N}(\mathscr{X}(\omega))}{N \varphi_{N}(\omega)} \Rightarrow N(0,1)
$$

as $N$ tends to infinity. And thus $S_{N}(\mathscr{X}(\omega)) /\left(\sqrt{2} \tau_{N}\right) \Rightarrow N(0,1)$ as $N$ tends to infinity, $\mathbf{P}$-a.s. Now we deduce from Remark 3.1 that $\mathbf{P}$-a.s.

$\lim _{j \rightarrow \infty} \sup _{N \in I_{j}}\left\|\frac{S_{N}(\mathscr{X})}{\tau_{N}}-\Theta_{j}\right\|_{2, m}=0 \Longrightarrow \lim _{j \rightarrow \infty} \sup _{N \in I_{j}}||\left|\frac{S_{N}(\mathscr{X})}{\tau_{N}}\left\|_{2, m}-\right\| \Theta_{j} \|_{2, m}\right|=0$.

But we have seen that

$$
\lim _{j \rightarrow \infty}\left\|\Theta_{j}\right\|_{2, m}^{2}=\lim _{j \rightarrow \infty} \frac{1}{b_{j}^{2}} \sum_{k>j}\left(g_{k}^{2}(\omega)+\left(g_{k}^{\prime}(\omega)\right)^{2}\right) \beta_{k}^{2}=2 \quad \text { P-a.s. }
$$

Thus $\mathbf{P}\left\{\lim _{N \rightarrow \infty}\left\|S_{N}(\mathscr{X}(\omega))\right\|_{2, m} / \tau_{N}=\sqrt{2}\right\}=1$. Finally,

$$
\frac{S_{N}(\mathscr{X}(\omega))}{\| S_{N}\left(\mathscr{X}(\omega) \|_{2, m}\right.} \Rightarrow N(0,1)
$$

as $N$ tends to infinity, $\mathbf{P}$-a.s. This achieves the proof.

We close this section by giving some examples.

Example 1. Put for $k \geqslant 3(\alpha>2, b>1): \varepsilon_{k}=k^{-\alpha / 2}, \beta_{k}=$ $\left(|k|(\ln |k|)^{b}\right)^{-1 / 2}$. 
Then $\prod_{k=2}^{n-1} \varepsilon_{k}^{-2}=\exp \left(\sum_{k=2}^{n-1} \alpha \ln k\right) \approx \exp \left(\alpha^{\prime} n \ln n\right)$; and thus

$$
N_{n}=\left\lfloor\frac{16^{n-1} N_{1}}{\varepsilon_{n} \varepsilon_{n-1}^{2} \cdots \varepsilon_{2}^{2} \varepsilon_{1}}\right\rfloor \approx \exp \left(\alpha^{\prime \prime} n \ln n\right),
$$

where $\alpha^{\prime}>0$ and $\alpha^{\prime \prime}>0$ depend on $\alpha$ only. Besides $b_{j}^{2} \approx$ $\sum_{|k| \geqslant j+1}|k|^{-1}(\ln |k|)^{-b} \approx(\ln j)^{-b+1}$. Further $\left(\beta_{j} / b_{j}\right) \sqrt{\ln j} \approx 1 / \sqrt{j} \rightarrow 0$ as $j$ tends to infinity. And

$$
\sum_{j \geqslant 1} \frac{\varepsilon_{j}^{2} \ln N_{j+1}}{b_{j}^{2}} \approx \sum_{j \geqslant 1} \frac{(\ln j)^{b-1} j \ln j}{j^{\alpha}}<\infty .
$$

Thus $\Delta_{r}<\infty$ for any $r>2$ (see (3.2)).

Consequently, $W_{r}(\mathscr{X}(\omega))<\infty$ and $\mathscr{X}(\omega) \in$ CLT for almost all $\omega \in \Omega$.

$\mathrm{Ex}$ a m p l e 2. Put for $k \geqslant 3(c>b>1): \varepsilon_{k}=\exp \left[-(\ln k)^{c}\right](k \geqslant 1)$, $\beta_{k}^{2}=\exp \left[-(\ln k)^{b}\right]-\exp \left[-(\ln (k+1))^{b}\right]$.

Then $\beta_{j}^{2} \approx(b / j)(\ln j)^{b-1} \exp \left[-(\ln j)^{b}\right]$. Hence $\beta_{j}^{2} / b_{j}^{2} \approx(b / j)(\ln j)^{b-1}$. Further, $\varepsilon_{n} \varepsilon_{n-1}^{2} \cdots \varepsilon_{2}^{2} \varepsilon_{1} \approx \exp \left[-n(\ln n)^{c}\right]$, and thus $N_{n} \approx \exp \left[-n(\ln n)^{c}\right]$. Therefore,

$$
\sum_{j \geqslant 1} \frac{\varepsilon_{j}^{2} \ln N_{j+1}}{b_{j}^{2}} \approx \sum_{j \geqslant 1} \exp \left[-(\ln j)^{c}+(\ln j)^{b}\right] j(\ln j)^{c}<\infty .
$$

Thus, $\Delta_{r}<\infty$ for any $r>2$.

Consequently, $W_{r}(\mathscr{X}(\omega))<\infty, \mathscr{X}(\omega)$ is continuous and $\mathscr{X}(\omega) \in \mathrm{CLT}$ for almost all $\omega \in \Omega$.

The third example is used in the next section.

$\mathrm{Ex}$ a m p l e 3 . Put for $k \geqslant 1: \beta_{k}^{2}=(\ln (k+2))^{-4} \exp \left[-k(\ln k)^{-4}\right]$, $\varepsilon_{k}^{2}=k^{-4} \exp \left[-k(\ln k)^{-4}\right]$.

The following technical lemma is elementary and based upon the fact that $\left(\exp \left[-x(\ln x)^{-4}\right]\right)^{\prime} \sim \exp \left[-x(\ln x)^{-4}\right](\ln x)^{-4}, x \rightarrow \infty$.

Define $d_{j}=\sum_{N_{j} \leqslant N<N_{j+1}} 1 / N, j \geqslant 1$.

\section{Lemma 5.2.}
a) $b_{j}^{2}:=\sum_{k \geqslant j} \beta_{k}^{2} \sim 2 \exp \left[-j(\ln j)^{-4}\right]$,
b) $N_{n} \sim \exp \left[n^{2}(\ln n)^{-4}\right]$,
c) $d_{j} \sim \ln N_{j}-\ln N_{j+1} \sim 2 j(\ln j)^{-4}$,
d) $\sum_{j \geqslant 1} \frac{\varepsilon_{j}}{\beta_{j}}\left(\ln N_{j+1}\right)^{1 / 2}<\infty$,
e) $\sum_{k \geqslant j}\left(\frac{\beta_{k}}{b_{j}}\right)^{3}(\ln k)^{3 / 2} \approx(\ln j)^{-1 / 2}$

Consequently $\mathscr{X}(\omega)$ is continuous and $\mathscr{X}(\omega) \in$ CLT for almost all $\omega \in \Omega$. 


\section{REFERENCES}

1. Berkes I. On almost i.i.d. subsequences of the trigonometric system. - Lecture Notes in Math., 1988, v. 1332, p. 54-63.

2. Bourgain J. Pointwise ergodic theorems for arithmetic sets. - Inst. Hautes Études Sci. Publ. Math., 1989, №69, p. 5-45.

3. Burton R., Denker $M$. On the central limit theorem for dynamical systems. - Trans. Amer. Math. Soc., 1987, v. 302, №2, p. 715-726.

4. Denker $M$. The central limit theorem for dynamical systems. - Banach Center Publ., 1986, v. 23, p. 33-62.

5. de la Rue T., Ladouceur S., Peskir G., Weber M. On the central limit theorem for aperiodic dynamical systems and applications. - Teor. İmovīr. Mat. Stat., 1997, № 57, p. 140-159.

6. Giuliano Antonini R., Weber M. The intersective ASCLT. - Stochastic Anal. Appl., 2004, v. 22, № 4, p. 1009-1025.

7. Giuliano Antonini R., Weber M. Counting occurrences in almost sure limit theorems. - Colloq. Math., 2005, v. 102, № 2, p. 271-290.

8. Gordin M., Weber $M$. On the almost sure central limit theorem for a class of $Z^{d}$-actions. - J. Theoret. Probab., 2002, v. 15, № 2, p. 477-501.

9. Gordin M., Weber M. A borderline Gaussian random Fourier series for the sampled convergence in variation. - J. Math. Anal. Appl. (to appear).

10. Kac $M$. On the distribution of values of sums of the type $\sum f\left(2^{k} t\right)$. - Ann. Math., 1946 , v. 47 , p. 33-49.

11. Kato Y. Central limit theorem for Weyl automorphism. - Rep. Statist. Appl. Res. Un. Japan. Sci. Engrs., 1987, v. 34, № 3, p. 1-10.

12. Lacey $M$. On central limit theorems, modulus of continuity and Diophantine type of irrational rotations. - J. Anal. Math., 1993, v. 61, p. 47-59.

13. Lacey M., Philipp W. A Note on the almost sure central limit theorem. - Statist. Probab. Lett., 1990, v. 9, p. 201-205.

14. Ledoux M., Talagrand M. Probability in Banach Spaces. Springer-Verlag, 1990, 480 p.

15. Mitrinovic D.S. Analytic Inequalities. New York-Berlin: Springer-Verlag, 1970, $400 \mathrm{p}$.

16. Olevskii A. M. Fourier Series with Respect to General Orthogonal Systems. New YorkHeidelberg: Springer-Verlag, 1975, 136 p.

17. Philipp W., Stout W. Almost sure invariance principles for partial sums of weakly dependent random variables. - Mem. Amer. Math. Soc., 1975, v. 161, issue 2, p. 1-140.

18. Salem R., Zygmund A. On lacunary trigonometric series. - Proc. Natl. Acad. Sci. USA, 1947, v. 33, p. 333-338.

19. Volny $D$. Invariance principles and Gaussian approximation for strictly stationary processes. - Trans. Amer. Math. Soc., 1999, v. 351, № 8, p. 3351-3371.

20. Weber $M$. Un théorème central limite presque sûr à moments généralisés pour les rotations irrationnelles. - Manuscripta Math., 2000, v. 101, № 2, p. 175-190.

21. Weber $M$. Entropie métrique et convergence presque partout. Paris: Hermann, 1998, $151 \mathrm{p}$.

Поступила в редакцию

10.IX.2003

Исправленный .вариант

29.III. 2005 